\title{
Research and Design of Mouse Scanner Module
}

\author{
Wen Long ${ }^{\mathrm{a}}$, Li Li, Weiqi Jin ${ }^{\mathrm{c}}$ \\ MOE Key Laboratory of Optoelectronic Imaging Technology and System, Beijing Institute of \\ Technology, Beijing 100081, China

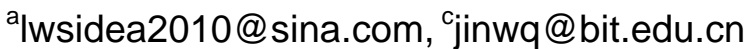

Keywords: Scanner module, Optical sensor, Embedded system, Image stitching.

\begin{abstract}
Compared with the ordinary books scanner's complexity and huge size, the design of a USB-based scanner module was much acceptable.In the module, the OV7670 was used for image acquisition, and two AVAGO optical sensors were used to acquire the position coordinates of the area which would be scanned. A frame of the image data stream of the region was transmitted to the PC side through the universal serial bus(USB) protocol, with the binding position information to solve the problem that recent similar device had high requirement on hardware performance, this simplified the equipment structure to reduce valume and costs. The module can get text or image information by scanning paper's surface in daily use.
\end{abstract}

\section{Introduction}

The emergence and development of the scanner has greatly promoted the digitization of books, which has greatly facilitated the preservation and transmission of the information, and has become the fast way of information translation. However, there are still many shortcomings in the current scanners, such as complex system structure with a simple function, and the annoying volume. Some special applications require minimal equipment volume, if the scanning part can be separated from the platform to optimizing the size, which is made a module and can be integrated into any platform, such as the smart cellphone.

James R. Montgomery applied for a scanner module patent based on two optical sensor, and gave us an overview of how the scanning module worked. Kenny Chan pointed out that the scanner module could be combined with the digital signal processor (DSP) platform. With the help of DSP on the device, the recovery of the scanned image was finished in the computer memory [1] .

LG has released an improved product the LSM100 based on microcontroller and personal computer, as shown in Figure 1. Its product module is relatively mature, however the volume is still need optimizing, it is regrettable that the official company does not provide any code, therefore there are no possibility to develop the module based on the this product. LG's product verify the feasibility of James R. Montgomery patent, thus we decided to follow the same direction as LG company, has completed the study of the module and the prototype of the production independently, finally the basic function has been realized in the module which can be applied to a variety of devices.

\section{Module design}

In James R. Montgomery's patent, the scanning module is equipped with a charge-coupled device (CCD) and two optical position sensors. The CCD is responsible for obtaining images, while the optical sensor is responsible for collecting the corresponding coordinates of the image.the use of two position sensors is to obtain the rotation angle of the image.

The scanning module in the project is integrated into the ordinary mouse. At the bottom of the mouse, a transparent glass window is opened as the image acquisition window, on both sides of the window, two optical position sensor distributed symmetrically. Image and position data are transmitted through the USB port. The implement of the scanning module consist of two parts: a microprocessor-based module function part and a working platform, as shown in Fig2. 
Cy7c68013A (FX2LP series) the MCU chip included an enhanced 8051 core, providing USB protocol serial interface engine (SIE) module which simplified the realization of the USB protocol[2]. As shown in Figure 2, the MCU combined the image data collected from the OV7670 and the location information obtained from ADNS-3060 together then sent it to the PC platform through the USB protocol for further image processing.
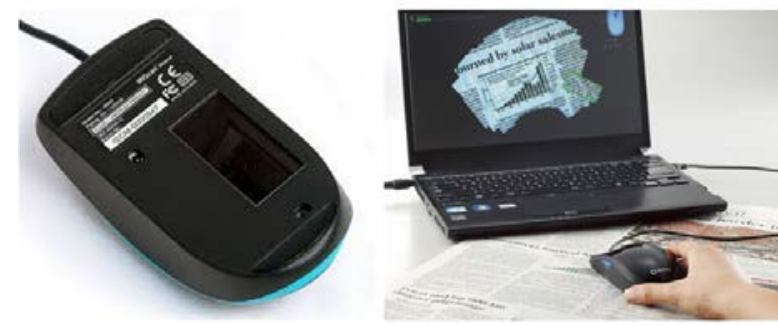

Fig.1 LG mouse scanner demonstration

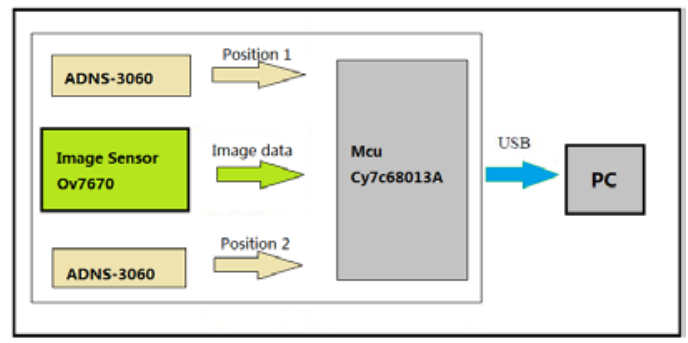

Fig.2 The designed module system structure

Circuit Design. OV7670 is a digital image acquisition device, which communicates with MCU through SCCB protocol and 8-bit wide data port. As shown in Figure 3, the image field signal VSYNC is connected to the external interrupt PA0 pin of the FX2LP. When the data of each frame comes, the processor informs the processor that the image data has arrived. The 8-bit data port (PB0 PB7) between The OV7670 and FX2LP are used for transmission of image data, and SCCB protocol port are used to control and initialize the device.

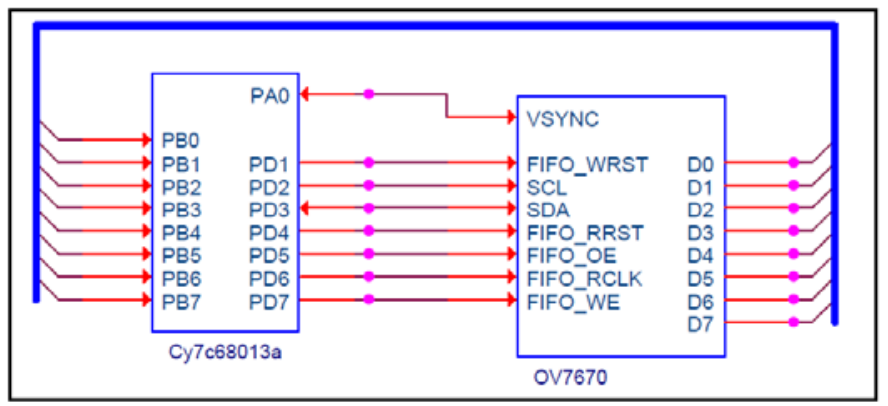

Fig.3 Connections of the image sensor

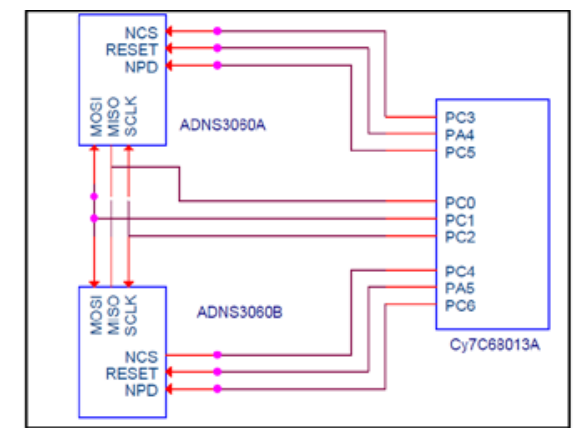

Fig.4 Connection of the Position Sensors

ADNS3060 the optical position sensor features a complete SPI four-wire port, which allows multiple sensors to share the SPI bus ,through which the MCU controls the sensor and acquires the position data. In figure 4, two ADNS3060 shared SPI bus (MOSI, MISO, SCLK) by choosing a different chip select signal NCS. For FX2LP without built-in SPI interface, the circuit used two IO port (PC0 PC2) to simulate the SPI protocol.

Firmware Design. As shown in Figure 5, the program waits for the start signal of the image acquisition from the PC after the completion of the hardware initialization. The image buffer output control is enabled at the beginning of the acquisition of the first frame image, simultaneously arm the position sensor. The datas of position sensors are accumulating, until the arrival of the second frame of the image. Thus the two frame images were obtained with their relative position.

The signal VSYNC of OV7670 is the field synchronization signal, which is active-low, the horizontal synchronization signal HREF is active-high. When the pin VSYNC is high, it indicates that one frame of data in the FIFO is ready. Data are buffered into the FIFO by the start of the VSYNC after data write enable signal FIFO_WE , the microcontroller opens the output enable signal FIFO_OE to read out this frame data and when the next VSYNC arriving reopen the write enable.The microcontroller reads the image data in bytes and transfers it to the internal high-speed FIFO, which is 512-byte depth twice buffer, and then send those to the PC .Due to the difference between the big endian and little endian ,the image data should be adjusted in little endian in firmware ,otherwise the saved image may not be displayed properly[3] . 


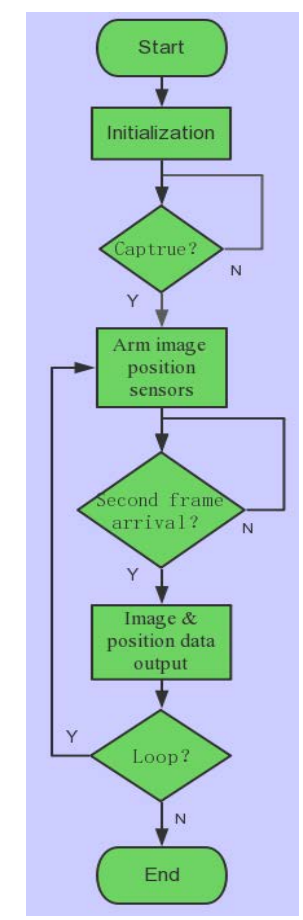

Fig. 5 Program flow chart

\section{Image Processing}

The driver Cypress provided meet the requirements well, while the new version of the CyAPI library simplifies the control of the hardware, so we do not need writing the driver code. The program interface was based on the Qt library, which realized the manual configuration of the image sensor, the real-time display of the acquired image (15FPS) and the recovery of the acquired image

Image stitching method. The movement in the plane of Scanning module can be equivalent to the superposition of translation and rotation[4] .As shown in Figure 6, the first three pictures were the image frames of the T-shaped patterns obtained by scanning in different translated positions, and the patterns were restored in computer memory according to the relative coordinates between them..
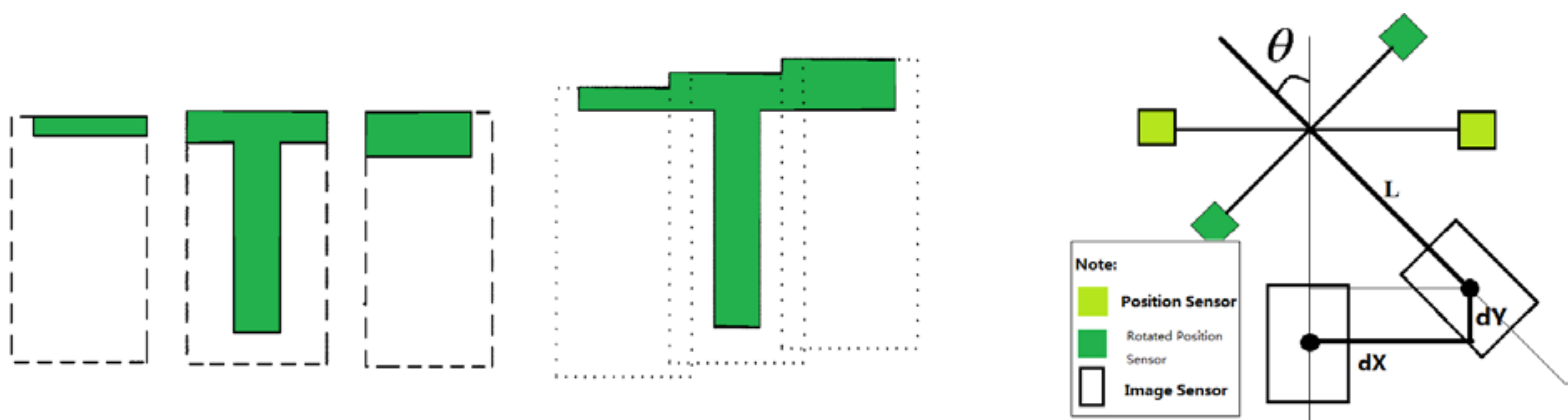

Fig.6 Image translation stitching

Fig.7 Rotating schematic diagram

The relative position and rotation angel between the image sensor and the two position sensors are shown in Figure 7,in which the vertical distance between image sensor and the optical sensor was defined as L. When the module was rotated only, the image sensor actually contained additional translation

$$
\begin{aligned}
& d X=L * \sin (\theta) \\
& d Y=-L * \cos (\theta)
\end{aligned}
$$


In formula $1, \mathrm{dX}$ is the horizontal additional movement, $\mathrm{dY}$ is the vertical additional movement in the formula 2, the negative sign indicate that the coordinate system is screen coordinate system, $\mathrm{Y}$ is increasing in down direction.

Final Result. As shown in Figure 8, the system test result comes out, in which A stands the card to be tested, $\mathrm{B}$ is the final output the system and $\mathrm{C}$ is the system user interface.

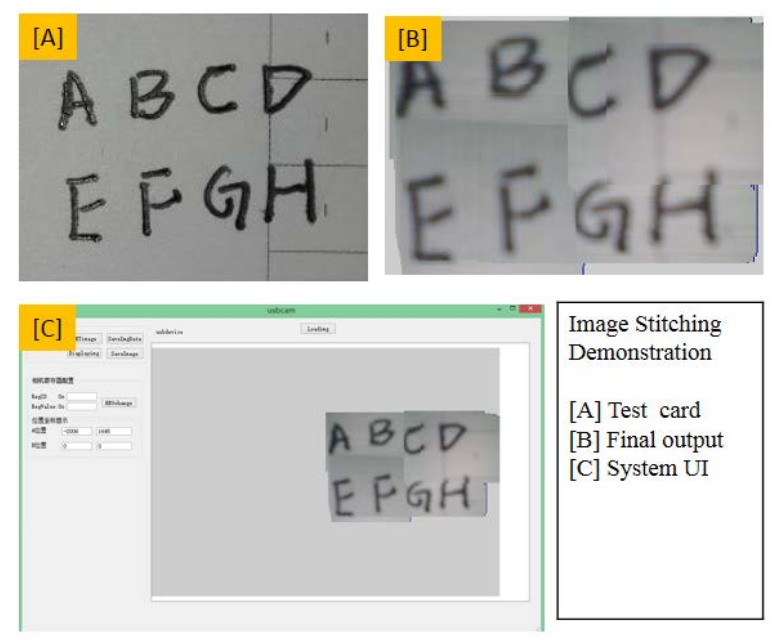

Fig.8 Final Output of the System and the Original Surface

In the figure 8 , the paper A with 8 characters was placed under the module just like what LG's product done, then the module was moved over the paper slowly.At the same time, the postion sensors and the image sensor at the bottom of the module got the data, and the MCU done the others through bulk transfer[5] .

\section{Summary}

The prototype of the module has realized the stitching and recovering method of the collected images with the corresponding positions, and the project code is finished and reliable. But the image still has small shortness, especially in the process of moving the scanner module when vertical position changed, this will lead to image dislocation. After further image processing and structural adjustment have been done for the module ,which can be applied to personal using and more wider fields which care about convenience and security.

\section{References}

[1] Kenny Chan. Mouse Scanner Position Display [P]: US. US20090244648 A1.2009

[2] Feng Qian. Programming Principle and Application of EZ-USB FX2 in Chinese [M].Beijing: Beihang University Press.2006

[3] Abramoff, M.D. Image processing with ImageJ [C].Biophotonics international, 2004.pp. 36 - 42

[4] Omnivision Technologies. systems and methods for resuming capture of a base image of an object by a mobile scanner: US.US 8,705,145 B2[P].2014

[5] Cypress Tech. Designing a Bulk Transfer Host Application for EZ-USB®FX2LPTM/FX3[EB/OL].2008.http://china.cypress.com/file/124291 